06,12

\title{
Размерный эффект в нанокомпозитах на основе молекулярного сегнетоэлектрика бромида диизопропиламмония
}

\author{
(C) C.В. Барышников ${ }^{1,2}$, А.Ю. Милинский ${ }^{1}$, Е.В. Чарная ${ }^{3}$, И.В. Егорова ${ }^{1}$ \\ ${ }^{1}$ Благовещенский государственный педагогический университет, \\ Благовещенск, Россия \\ ${ }^{2}$ Амурский государственный университет, \\ Благовещенск, Россия \\ ${ }^{3}$ Санкт-Петербургский государственный университет, \\ Санкт-Петербург, Россия \\ E-mail: svbar2003@list.ru
}

(Поступила в Редакцию 25 июня 2018 г.

В окончательной редакции 14 августа 2018 г.)

\begin{abstract}
Представлены результаты исследований диэлектрических свойств нанокомпозитов на основе оксидных пленок $\mathrm{Al}_{2} \mathrm{O}_{3}$ с диаметром пор 330 и $60 \mathrm{~nm}$ с введенными в поры частицами органического сегнетоэлектрика бромида диизопропиламмония $\left(\mathrm{C}_{6} \mathrm{H}_{16} \mathrm{BrN}\right.$, DIPAB), проведенных с целью выявления размерных зависимостей параметров фазового перехода. Обнаружено смещение фазового перехода к низким температурам и размытие перехода, которые становятся более значительными для пор меньшего размера. Для нанокомпозитов наблюдалось также уширение температурного гистерезиса диэлектрической проницаемости при фазовом переходе. Понижение температуры фазового перехода в нанокомпозитах с наночастицами DIPAB согласуется с теоретическими моделями влияния размерных эффектов на структурный фазовый переход.
\end{abstract}

DOI: 10.21883/FTT.2019.02.47125.168

\section{1. Введение}

Молекулярные сегнетоэлектрики относятся к молекулярным кристаллам [1], претерпевающим структурный фазовый переход в одну из полярных групп и обладающим сегнетоэлектрическими свойствами [2,3]. Молекулярные сегнетоэлектрики имеют ряд значительных преимуществ по сравнению с широко используемыми неорганическими сегнетоэлектриками, таких как экологическая безопасность, поскольку они не содержат тяжелых металлов, и малый вес. Однако применение молекулярных сегнетоэлектриков было ограничено их небольшой спонтанной поляризацией, слабым пьезоэлектрическим эффектом и низкими температурой Кюри и температурой плавления. Эти ограничения снимаются открытиями последних лет, когда были обнаружены органические соединения с высокой спонтанной поляризацией, обладающие сегнетоэлектрическими свойствами при комнатной температуре и имеющие достаточно высокую температуру плавления. К ним относятся хлорид диизопропиламмония (DIPAC) со спонтанной поляризацией при комнатной температуре $P_{s} \sim 8.2 \mu \mathrm{C} \cdot \mathrm{cm}^{-2}$ и температурой Кюри $T_{c}=440 \mathrm{~K}$ [4] и бромид диизопропиламмония (DIPAB) с $P_{s} \sim 23 \mu \mathrm{C} \cdot \mathrm{cm}^{-2}$ и $T_{c}=426 \mathrm{~K}$ [5]. Относительно третьего соединения из этой группы, иодида диизопропиламмония (DIPAI), в литературе имеются противоречивые сведения [6,7], но недавно для DIPAI были измерены спонтанная поляризация $P_{x} \sim 33 \mu \mathrm{C} \cdot \mathrm{cm}^{-2}$ и $T_{c}=415 \mathrm{~K}[8]$. Благодаря высоким значениям спонтанной поляризации и температуры Кюри галогениды диизопропиламмония могут найти разнообразные практические применения. Современный тренд в приклад- ной физике сегнетоэлектриков состоит в разработке электронных устройств с низкоразмерными элементами - тонкими пленками, нанонитями и малыми частицами $[9,10]$. Нанокомпозиты на основе мезопористых матриц, в поры которых введены сегнетоэлектрические частицы, рассматриваются как модельные системы для изучения свойств систем пониженной размерности, а также представляют самостоятельный интерес для возможных применений. Центральным вопросом при анализе свойств сегнетоэлектрических нанокомпозитов на основе пористых матриц является выяснение размерных зависимостей характеристик структурного фазового перехода и, прежде всего, возможности существования фазового перехода для материалов в порах. Для молекулярных сегнетоэлектриков галогенидов диизопропиламмония имеется только одна работа такого рода, в которой изучалось смещение температуры сегнетоэлектрического перехода для DIPAC в порах опаловой матрицы и молекулярных сит МСМ-41 [11]. Отметим, что в работе [12] проводились исследования тонких пленок DIPAB.

В настоящей работе приводятся результаты исследований комплексной диэлектрической проницаемости нанокомпозитов на основе пористых пленок оксида алюминия $\mathrm{Al}_{2} \mathrm{O}_{3}$ с диаметром пор 330 и $60 \mathrm{~nm}$ с внедренным в поры бромидом диизопропиламмония, DIPAB. Для сравнения проводились также диэлектрические измерения объемных образцов DIPAB.

\section{2. Образцы и эксперимент}

DIPAB при комнатной температуре может существовать в двух различных полиморфных фазах с простран- 
ственной симметрией $P 22_{1}$ или $P 2{ }_{1} 2{ }_{1} 2_{1}$, в зависимости от условий получения и термальной истории $[5,13-$ 15]. Моноклинная фаза с симметрией $P 2_{1}$ является сегнетоэлектрической. При температуре около $425 \mathrm{~K}$ она претерпевает структурный переход в неполярную моноклинную фазу с симметрией $P 2_{1} / m$. Сегнетоэлектрический переход в DIPAB относится к переходам первого рода $[5,14]$. Вторая стабильная при комнатной температуре фаза имеет ромбическую симметрию с пространственной группой $P 2{ }_{1} 2_{1} 2_{1}$ и сегнетоэлектрически неактивна. При нагреве она также переходит в неполярную моноклинную фазу $P 2_{1} / \mathrm{m}$, но с образованием промежуточной полярной структуры с симметрией $P 2_{1}$, которая существует в интервале от примерно 421 до $425 \mathrm{~K}$. При охлаждении от температуры выше $425 \mathrm{~K}$ ромбическая фаза больше не образуется.

В наших исследованиях бромид диизопропиламмония был получен реакцией диизопропиламина с 48\%м водным раствором $\mathrm{HBr}$ (эквимолярное соотношение) по методике, приведенной в [13], с последующей перекристаллизацией в метиловом спирте при комнатной температуре. Согласно работе [13] такая методика приводит к росту кристаллов, имеющих моноклинную симметрию $P 2_{1}$. Максимальные кристаллики имели размеры $2-3 \mathrm{~mm}$. Для диэлектрических измерений в качестве объемных образцов брались таблетки диаметром $12 \mathrm{~mm}$ и толщиной около $1.5 \mathrm{~mm}$, полученные прессовкой кристаллического порошка $\mathrm{C}_{6} \mathrm{H}_{16} \mathrm{BrN}$ под давлением $6000-7000 \mathrm{~kg} / \mathrm{cm}^{2}$. Снимок поверхности таблетки показан на рис. 1. Для получения нанокомпозитов использовались оксидные пленки $\mathrm{Al}_{2} \mathrm{O}_{3}$, изготовленные фирмой „ТорMembranes Technology“ (China), с размерами ячеек 450 и $100 \mathrm{~nm}$ и диаметрами пор 330 и $60 \mathrm{~nm}$ соответственно. Глубина пор составляла около $50 \mu \mathrm{m}$. Внедрение сегнетоэлектрика в поры производилось из нагретого насыщенного раствора $\mathrm{C}_{6} \mathrm{H}_{16} \mathrm{BrN}$ в метаноле. Пленка помещалась в раствор,

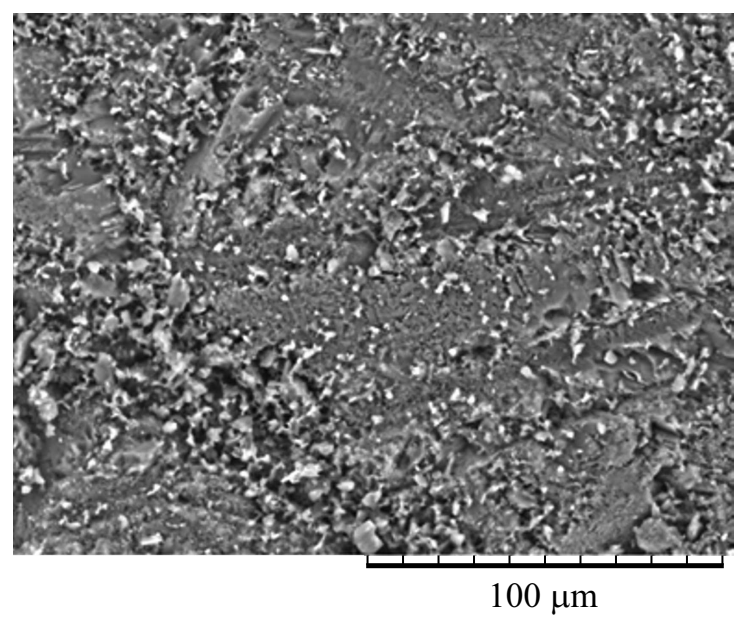

Рис. 1. Электронное изображение поверхности прессованного образца.
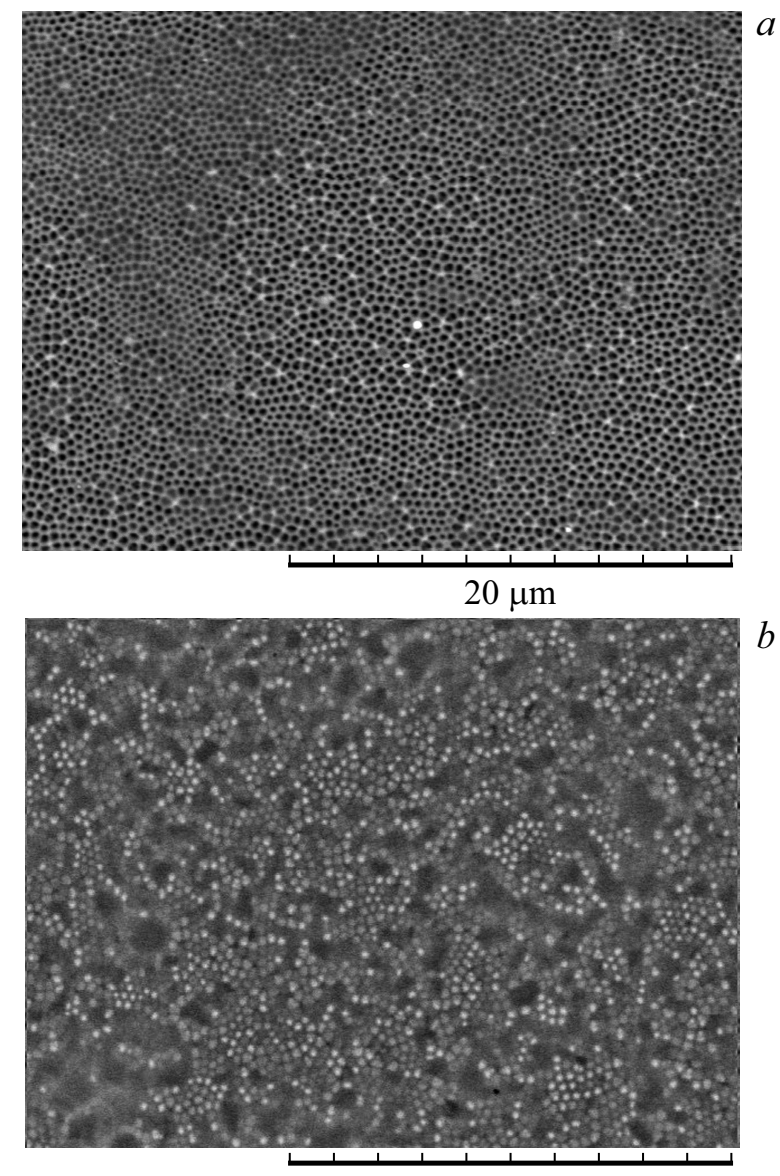

$20 \mu \mathrm{m}$

Рис. 2. Электронный снимок пленки $\mathrm{Al}_{2} \mathrm{O}_{3}$ с порами $330 \mathrm{~nm}$ до $(a)$ и после $(b)$ внедрения DIPAB.

который медленно охлаждался, в результате чего в порах зарождались нанокристаллы, растущие в течение нескольких дней. После трехкратного повторения описанной процедуры поры заполнялись. Для удаления оставшегося метанола использовалась вакуумная сушка. Степень заполнения пор, определенная по изменению массы пленок, составляла не менее $60 \%$. Полученные образцы можно рассматривать как систему параллельных наностержней DIPAB. Электронные снимки пустой и заполненной пленок $\mathrm{Al}_{2} \mathrm{O}_{3}$ показаны на рис. 2.

Для измерения комплексной диэлектрической проницаемости использовался цифровой измеритель иммитанса E7-25 с частотным диапазоном $20 \mathrm{~Hz}-1 \mathrm{MHz}$ и рабочим напряжением $0.7 \mathrm{~V}$. В качестве электродов использовалась In-Ga паста. Температура определялась с помощью электронного термометра ТС-6621 с хромель-алюмелевой термопарой. Точность определения температуры составляла $0.1 \mathrm{~K}$. Измерения проводились в режиме нагрева и последующего охлаждения со скоростью $1 \mathrm{~K}$ в минуту в температурном интервале от 300 до 450 К. Погрешность измерения диэлектрической проницаемости не превышала 5\%. 


\section{3. Экспериментальные результаты и обсуждение}

Зависимости реальной части диэлектрической проницаемости $\varepsilon^{\prime}$ и тангенса угла диэлектрических потерь $\tan \delta$ от температуры для объемных образцов DIPAB, полученные в режиме нагрева на нескольких частотах, представлены на рис. 3. Максимумы на кривых $\varepsilon^{\prime}(T)$ наблюдаются при температуре $425.8 \mathrm{~K}$, что соответствует структурному переходу $P 2_{1} \rightarrow P 2_{1} / m$ и согласуется с результатами, представленными в работах [5,13-15]. Сильная дисперсия $\varepsilon^{\prime}$ и $\tan \delta$ также соответствует опубликованным данным для объемного DIPAB. При охлаждении максимум вещественной части диэлектрической проницаемости сдвигается в сторону низких температур примерно на 1 градус. Температурный гистерезис согласуется с первым родом фазового перехода.

На рис. 4 представлены зависимости вещественной части диэлектрической проницаемости $\varepsilon^{\prime}$ и тангенса угла диэлектрических потерь $\tan \delta$ от температуры для заполненных пленок $\mathrm{Al}_{2} \mathrm{O}_{3}$, полученные в режиме нагрева. Как видно на рис. 4 , сильная дисперсия диэлектрических характеристик сохраняется и для нанокомпозитов с частицами DIPAB. Диэлектрические аномалии в области фазового перехода для нанокомпозитов менее выражены по сравнению с объемными образцами. Фазовому переходу соответствуют размытые изломы на кривых $\varepsilon^{\prime}(T)$, которые становятся заметнее с понижением частоты. Большие значения вещественной части диэлектриче-

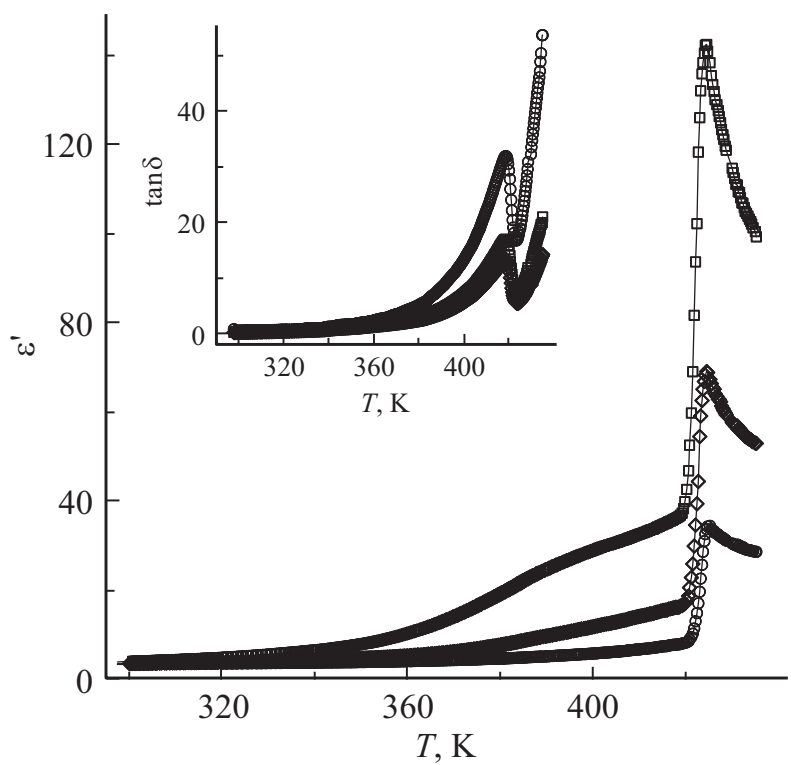

Рис. 3. Температурные зависимости вещественной части диэлектрической проницаемости для прессованного поликристаллического образца DIPAB, полученные при нагреве на частотах $1 \mathrm{kHz}$ (квадраты), $10 \mathrm{kHz}$ (ромбы) и $100 \mathrm{kHz}$ (кружки). На вставке представлены температурные зависимости тангенса угла потерь, полученные на тех же частотах. Символы на вставке соответствуют обозначением на основном рисунке.
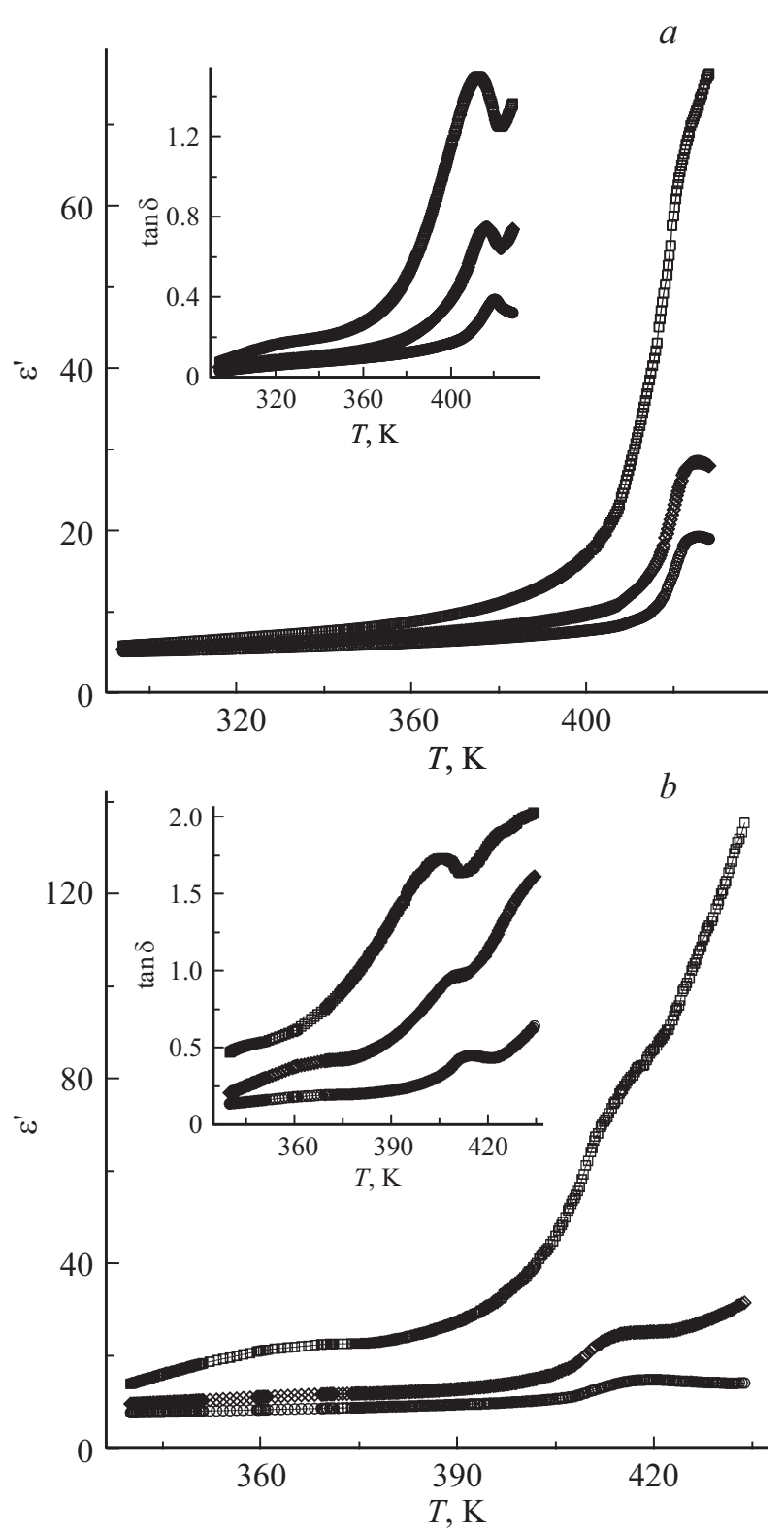

Рис. 4. Температурные зависимости вещественной части диэлектрической проницаемости для заполненных DIPAB пленок $\mathrm{Al}_{2} \mathrm{O}_{3}$ с порами $330 \mathrm{~nm}(a)$ и $60 \mathrm{~nm}(b)$, полученные при нагреве на частотах $1 \mathrm{kHz}$ (квадраты), $10 \mathrm{kHz}$ (ромбы) и $100 \mathrm{kHz}$ (кружки). На вставках представлены температурные зависимости тангенса угла потерь, полученные на тех же частотах. Символы на вставках соответствуют обозначением на основных рисунках.

ской проницаемости в нанокомпозитах выше области фазового перехода, по-видимому, обусловлены вкладом поляризации Максвелла-Вагнера [16], которая возникает за счет перераспределения зарядовой плотности на границах раздела матрицы и включений DIPAB.

Для сравнения температур структурных переходов в объемном образце и нанокомпозитах, степени размытия фазовых переходов и величины температурного гистерезиса на рис. 5 показаны температурные зависимости 


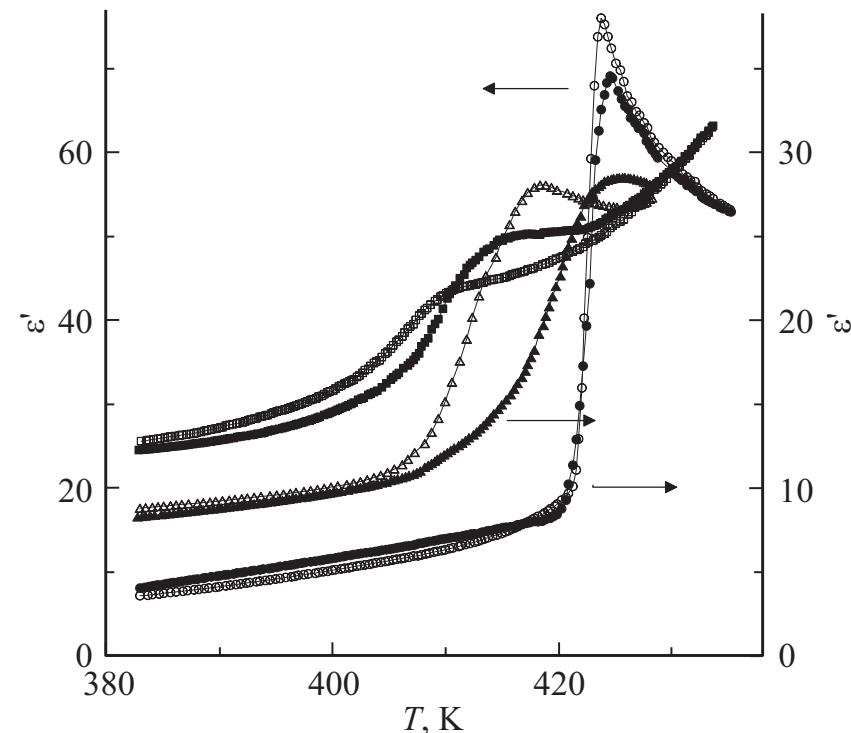

Рис. 5. Температурные зависимости вещественной части диэлектрической проницаемости для прессованного поликристаллического образца DIPAB (кружки, левая ось) и заполненных DIPAB пленок $\mathrm{Al}_{2} \mathrm{O}_{3}$ с порами $330 \mathrm{~nm}$ (треугольники, правая ось) и $60 \mathrm{~nm}$ (квадраты, правая ось), полученные при нагреве (темные символы) и охлаждении (светлые символы) на частоте $10 \mathrm{kHz}$.

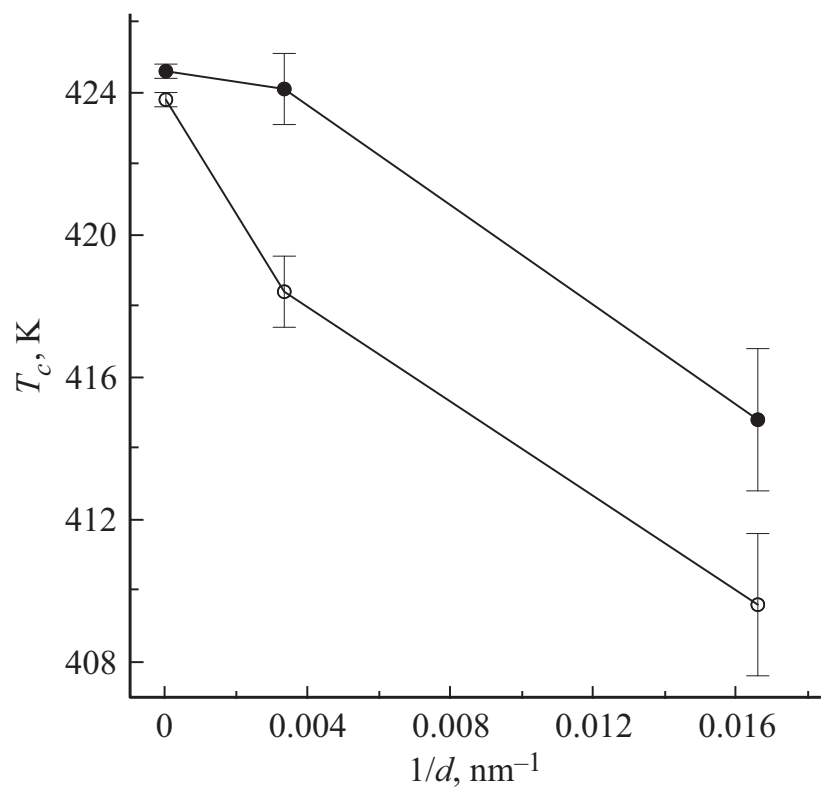

Pис. 6. Температура сегнетоэлектрического фазового перехода при нагреве (темные символы) и охлаждении (светлые символы) в зависимости от обратного размера пор $d$. Объемному образцу соответствует $1 / d=0$.

вещественной части диэлектрической проницаемости, полученные в режимах нагрева и охлаждения на частоте $10 \mathrm{kHz}$. Из графиков на рис. 5 видно, что связанные с сегнетоэлектрическим фазовым переходом аномалии диэлектрической проницаемости в нанокомпозитах сдви- гаются в сторону низких температур по сравнению с объемным образцом. При этом сдвиг увеличивается с уменьшением диаметра пор. Зависимость температур $T_{c}$ сегнетоэлектрического перехода, наблюдаемого при нагреве и охлаждении, от размера пор представлена на рис. 6. Отметим также, что, наряду с понижением температурных интервалов фазовых переходов и значительным размытием переходов, в нанокомпозитах уширяется температурный гистерезис, что свидетельствует об усилении степени первородности в условиях нанокомнфайнмента.

Результаты по изменению температуры фазового перехода, представленные на рис. 6, можно сравнить с аналогичными результатами, полученными для сегнетовой соли и нитрита натрия, введенных в пористый оксид алюминия [17-20]. Для сегнетовой соли в пористом $\mathrm{Al}_{2} \mathrm{O}_{3}$ наблюдалось расширение области существования сегнетоэлектрической фазы за счет понижения температуры нижнего структурного перехода и повышения температуры верхнего структурного перехода выше температуры разложения [17-19]. Для нитрита натрия было обнаружено повышение температуры структурного фазового перехода при введении в пористый алюминий, тогда как для нанокомпозитов на основе силикатных матриц с наночастицами нитрита натрия температура перехода понижалась [20]. В настоящей работе сегнетоэлектрический фазовый переход для наночастиц DIPAB в пористом оксиде алюминия сдвигался к низким температурам. Можно предположить, что разный знак сдвига температуры перехода для перечисленных выше сегнетоэлектриков обусловлен доминированием различных приводящих к сдвигу механизмов. При интерпретации смещения фазового перехода по температуре для наночастиц в условиях наноконфайнмента, как правило, используются модели размерных эффектов, разработанные для изолированных частиц на основе феноменологической теории Ландау и модели Изинга $[21,22]$. Эти модели предсказывают, что температура структурного фазового перехода должна понижаться при уменьшении размеров частиц, если параметр порядка или величина обменного интеграла на границах частиц меньше, чем в объеме. Выводы этих моделей были экспериментально подтверждены для отдельных малых частиц сегнетоэлектриков типа титаната бария (см. [23] и ссылки в этой работе). Однако для частиц в мезопористых матрицах к сдвигу температуры фазового перехода может приводить также взаимодействие со стенками пор и взаимодействие между частицами в соседних порах [19,20,24,25]. В работе [17] повышение температуры верхнего фазового перехода в наночастицах сегнетовой соли, выращенных в пористом оксиде алюминия, приписывалось поляризации стенок пор. В рамках модели размерных эффектов, основанной на теории Ландау, это соответствует росту спонтанной поляризации на поверхности частиц и, как следствие, сдвигу фазового перехода к высоким температурам [21]. Кроме того, на сдвиге фазового перехода может сказываться электрическое взаимодействие 
между сегнетоэлектрическими частицами в порах [25]. Причем в зависимости от геометрии сетки пор и формы частиц такое взаимодействие может изменять влияние размерных эффектов, приводя как к повышению, так и понижению температуры перехода.

Для пористого оксида алюминия значительные размеры ячеек препятствуют установлению сильного взаимодействия между частицами в порах. Таким образом, следует предположить, что сдвиг температуры фазового перехода должен, в основном, определяться тем, насколько значительную роль играет поляризация стенок пор, заполненных конкретным сегнетоэлектриком. Для случая DIPAB в пористом оксиде алюминия поляризация, повидимому, незначительна и температура перехода понижается, как и для отдельной изолированной частицы. Размытие фазового перехода может являться следствием разброса размеров частиц в порах и различия форм частиц из-за неполного заполнения пор.

Представляет отдельный интерес увеличение ширины температурного гистерезиса для нанокомпозитов с DIPAB. B объемных сегнетоэлектриках изменение рода структурного фазового перехода или степени его удаления от трикритической точки вызывается повышением давления, а также изменением концентрации компонент твердых растворов [26]. Для нанокомпозитов с частицами DIPAB причиной усиления степени первородности фазового перехода, возможно, являются механические напряжения, развивающиеся в условиях наноконфайнмента.

\section{4. Заключение}

Таким образом, исследования пористых оксидных пленок $\mathrm{Al}_{2} \mathrm{O}_{3}$ с введенными в поры наночастицами молекулярного сегнетоэлектрика DIPAB, проведенные методами диэлектрической спектроскопии, выявили смещение сегнетоэлектрического фазового перехода к низким температурам по сравнению с объемным DIPAB, увеличивающееся с уменьшением размера пор. Для DIPAB в порах наблюдалось также размытие фазового перехода и увеличение температурного гистерезиса диэлектрической проницаемости. Предположено, что понижение температуры перехода, согласующееся с известными теоретическими моделями для изолированных малых сегнетоэлектрических частиц, свидетельствует о незначительной роли поляризации стенок матрицы.

\section{Список литературы}

[1] А.И. Китайгородский. Молекулярные кристаллы. Наука, M. (1971). 424 c.

[2] M. Owczarek, K.A. Hujsak, D.P. Ferris, A. Prokofjevs, I. Mayerz, P. Szkalarz, H. Zhang, A.A. Sarjean, C.L. Stern, R. Jakubas, S. Hong, V.P. Dravid, J.F. Stoddart. Nature Commun. 7, 13108 (2016).

[3] S. Horiuchi, Y. Tokura. Nature Mater. 7, 357 (2008).
[4] D.-W. Fu, W. Zhang, H.-L. Cai, J.-Z. Ge, Y. Zhang, R.G. Xiong. Adv. Mater. 23, 5658 (2011).

[5] D.-W. Fu, H.-L. Cai, Y. Liu, Q. Ye, W. Zhang, Y. Zhang, X.-Y. Chen, G. Giovannetti, M. Capone, J. Li, R.-G. Xiong. Science 339, 425 (2013).

[6] R.K. Saripalli, D. Swain, S. Prasad, H. Nhalil, H.L. Bhat, T.N.G. Row, S. Elizabeth. J. Appl. Phys. 121, 114101 (2017).

[7] A. Piecha-Bisiorek, A. Ga̧gor, D. Isakov, P. Zielinsski, M. Gałązka, R. Jakubas. Inorg. Chem. Front. 4, 553 (2017).

[8] E. Kabir, M. Khatun, T. Ghosh, M.J. Raihan, M. Rahman. AIP Conf. Proc. 1942, 040006 (2018).

[9] Nanoscale ferroelectrics and multiferroics: key processing and characterization issues, and nanoscale effects / Eds M. Alguero, J.M. Gregg, L. Mitoseriu. John Wiley \& Sons, Chichester, UK (2016).

[10] J.F. Scott, F.D. Morrison, M. Miyake, P. Zubko. Ferroelectrics 336, 237 (2006).

[11] S.V. Baryshnikov, E.V. Charnaya, A.Yu. Milinskiy, V.A. Parfenov, I.V. Egorova. Phase Transitions 91, 293 (2018).

[12] C. Thirmal, P.P. Biswas, Y.J. Shin, T.W. Noh, N.V. Giridharan, A. Venimadhav, P. Murugavel. J. Appl. Phys. 120, 124107 (2016).

[13] A. Piecha, A. Ga̧gor, R. Jakubasa, P. Szklarz. Cryst. Eng. Commun. 15, 940 (2013).

[14] Y. Li, K. Li, J. He. Chem. Phys. Lett. 689, 174 (2017).

[15] H. Yadav, N. Sinha, S. Goel, A. Hussain, B. Kumar. J. Appl. Cryst. 49, 2053 (2016).

[16] K.W. Wagner. Die Isolierstoffe der Elektrotechnik. Springer, Berlin (1924).

[17] D. Yadlovker, S. Berger. Phys. Rev. B 71, 184112 (2005).

[18] S.V. Baryshnikov, E.V. Charnaya, E.V. Stukova, A.Yu. Milinskiy, C. Tien. Ferroelectrics 396, 3 (2010).

[19] С.В. Барышников, Е.В. Чарная, Е.В. Стукова, А.Ю. Милинский, С. Tien. ФТТ 52, 1347 (2010).

[20] C. Tien, E.V. Charnaya, D.Yu. Podorozhkin, M.K. Lee, S.V. Baryshnikov. Phys. Status Solidi B 246, 2346 (2009).

[21] W.L. Zhong, Y.G. Wang, P.L. Zhang, B.D. Qu. Phys. Rev. B 50, 698 (1994)

[22] C.L. Wang, Y. Xin, X.S. Wang, W.L. Zhong. Phys. Rev. B 62, 11423 (2000).

[23] P. Sedykh, D. Michel. Phys. Rev. B 79, 134119 (2009).

[24] X.Y. Lang, Q. Jiang. J. Nanoparticle Res. 9, 595 (2007).

[25] A.V. Uskov, E.V. Charnaya, A.L. Pirozerskii, A.S. Bugaev. Ferroelectrics 482, 70 (2015).

[26] М. Лайнс, А. Гласс. Сегнетоэлектрики и родственные им материалы. Мир, М. (1981). 736 с.

Редактор Ю.Э. Китаев 